\title{
The effect of unreinforced-reinforced sequences on resistance to extinction following partial reinforcement'
}

\author{
RONALD L. KOTESKEY, ${ }^{2}$ Wayne State University, Detroit, \\ Mich. 48202
}

$A$ previous experiment had pointed to the number of unreinforced-reinforced (UR) sequences as the crucial variable in determining the magnitude of the increased resistance to extinction following partial reinforcement, called the partial reinforcement effect (PRE). Fifty albino rats were the Ss. $A 2$ by 2 factorial design was used with number of UR sequences as one factor; percentage, number, and length of run of nonreinforcements were confounded as the other factor. The magnitude of the PRE was not a function of UR sequences but it increased as the percentage, number, and length of run of nonreinforcements increased.

Several attempts have been made to determine what variable is responsible for the increased resistance to extinction following partial reinforcement, commonly called the partial reinforcement effect (PRE). Early experiments seemed to show an inverse relationship between percentage of reinforcement and resistance to extinction. Lewis (1960) concluded that there was an inverted $U$ relationship between these variables with low resistance to extinction at very high or very low percentages of reinforcement and increased resistance to extinction at the middle percentages of reinforcement.

Lawrence \& Festinger (1962) showed that the variable responsible for increased resistance to extinction was not percentage of reinforcement itself, but the number of nonreinforced responses experienced by each animal which had been confounded with percentage of reinforcement in earlier experiments. Grosslight \& Radlow (1957) had found that for the increased resistance to extinction to be found the nonreinforcements must be followed by a reinforcement within a day's block of trials.

Koteskey \& Stettner (1968) reasoned that in increasing the number of nonreinforcements, the number of unreinforcedreinforced (UR) sequences would also be increased and this may be the variable which is responsible for the magnitude of the PRE. They concluded, by elimination, that the crucial variable was the number of UR sequences. In groups where the number of UR sequences was equal, resistance to extinction was equal despite differences in percentage, number and length of run of nonreinforcements. In those experiments number of UR sequences was held constant with the other variables run at different levels. The following experiment attempted to show that by varying the number of UR sequences, different amounts of resistance to extinction would be found. Percentage, number, and length of run of nonreinforcements were all confounded and will be referred to as number of nonreinforcements.

\section{DESIGN}

A 2 by 2 factorial design was used with number of UR sequences and number of nonreinforcements as the two factors. The two values used for number of nonreinforcements were 31 and 40; the values used for number of UR sequences were 13 and 22 . A control group receiving continuous reinforcement was also used.

\section{SUBJECTS}

The Ss were 50 naive male albino rats of the Sprague-Dawley strain. Ten animals were randomly assigned to each group.

\section{APPARATUS}

An 8-ft straight alley fully described in Koteskey \& Stettner (1968) was used. Reinforcement again consisted of $180 \mathrm{mg}$ of food placed in the food cup by $E$.

\section{PROCEDURE}

The Ss were put on food deprivation and maintained on a 10-g-per-day feeding schedule throughout the course of the experiment. They were handled each day during pretraining and fed once in the goal box with the timers plugged in to become accustomed to the slight noise of the relays.

On each of the first two days of acquisition they were given three reinforced trials, then put on the schedules of reinforcement shown in Table 1 for nine days. The Ss were given the schedules in order so that schedule " $A$ " was used three times and each of the other schedules (B, C, and D) was used twice. A total of 77 acquisition trials was given. The trials were run with a 15-min intertrial interval and the Ss remained in the goal box $15 \mathrm{sec}$, more if necessary to finish eating. The Ss were fed their daily ration of $10 \mathrm{~g}$ of food $15 \mathrm{~min}$ after the last trial of the day.

Extinction began on Day 12 and consisted of seven nonreinforced trials each day with a 15 -min intertrial interval. Ss were run until they reached a criterion of two trials over $\mathbf{6 0}$ sec.

\section{RESULTS}

At the end of the acquisition period the running speed of all groups was not significantly different. Table 1 also shows the mean number of extinction trials to reach the criterion of two trials over $60 \mathrm{sec}$. The control group extinguished in 26.3 trials. Analysis of variance showed that the only significant variable was number of nonreinforcements, $F(1,36)=7.39$, $\mathrm{p}<.01$; the number of UR sequences and interaction yielded $\mathrm{F}<1$. A comparison of the control group with all other groups yielded $F(1,45)=23.39, p<.01$, so the experimental groups were more resistant to extinction than the control group.

\section{DISCUSSION}

This experiment was conducted under the same conditions as the Koteskey \& Stettner (1968) experiments, so the results

Table 1

Daily Acquisition Schedules of Reinforcement and Mean Trials to Extinction for Each of the Experimental Groups

\begin{tabular}{|c|c|c|c|c|}
\hline \multicolumn{3}{|c|}{40 Nonreinforcements } & \multicolumn{2}{|c|}{31 Nonreinforcements } \\
\hline Schedule & 22 UR Sequences & 13 UR Sequences & 22 UR Sequences & 13 UR Sequences \\
\hline A & RUURUUR & RRUUUUR & RUURRUR & RRUUURR \\
\hline B & UURRUUR & URRUUUR & UURRURR & URRRUUR \\
\hline $\mathrm{C}$ & RUURURUUR & RRUUUUURR & RURUURRUR & RRUUUURRR \\
\hline D & URRUURUUR & UURRUUURR & URRURUURR & UURRRRUUR \\
\hline
\end{tabular}

Mean Trials

to Extinction

43.0

45.4

36.8

37.8 


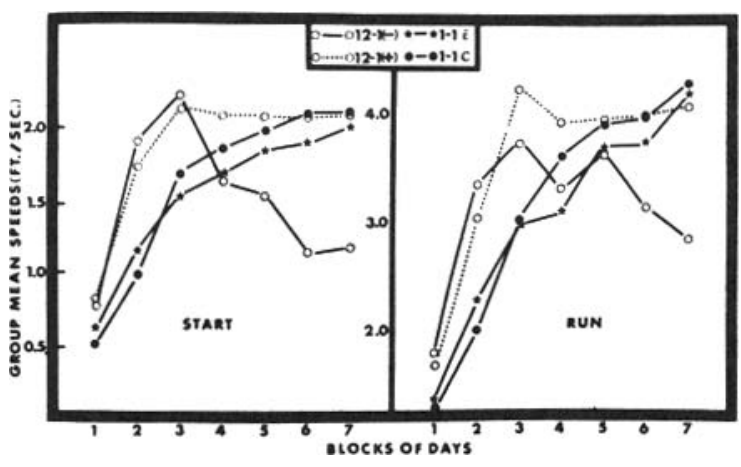

Fig. 1. Group mean start and run speeds for the various conditions as a function of blocks of two daily sessions.

differences between either the start or run speed means for Groups $\mathrm{E}$ and $\mathrm{C}$. However, Group $\mathrm{C}$ speeds were significantly faster than Group D speeds in both start and run measures $(p<.05)$. Group E speeds were significantly faster than Group D speeds in the start measure $(p<.05)$ and differed only slightly less significantly in the run measure $(p<.075)$. Finally, inspection of Fig. 1 indicates that Group D did form a discrimination, running faster on + than on - trials in the later stages of training. Analysis of variance of the data for Group D over Blocks 6-7 yielded a significant discrimination effect in both start and nun measures, $F=7.97$, $\mathrm{df}=1 / 6, \mathrm{p}<.05$ and $\mathrm{F}=8.51, \mathrm{df}=1 / 6, \mathrm{p}<.05$, respectively.

\section{DISCUSSION}

Considering the results of the present study with respect to speeds on one-pellet ( $\mathrm{S}-$ ) trials in the runway, at least two observations warrant attention. First, Group D, the discrimination group, ran significantly slower on one-pellet trials than did Group C. This finding is highly similar to the general phenomenon of negative $\mathrm{S}$ - contrast. Secondly, the performance levels of Groups $E$ and $C$ did not differ on one-pellet trials in the runway even though Group $\mathrm{E}$ received large (12-pellet) reward experience and Group $C$ did not. Taken jointly, these two findings demonstrate that depression of speeds to a stimulus associated with the smaller of two magnitudes of reward is not due merely to experience with larger reward magnitudes since both Groups $D$ and $E$ experienced large reward. Rather, these observations suggest that conditions under which the larger reward is experienced is important in producing a depression of $\mathrm{S}-$ speeds.

The findings of the present study, in conjunction with previous literature, indicate that merely receiving larger magnitude of reward is not sufficient to produce negative $S-$ contrast, but that contrast is produced when larger rewards are contingently experienced in the S+ runway. This indication seems relevant to the theoretical dichotomy made by Dunham (1968) between perceptual-motivational and emotionalmotivational interpretations of contrast. Generally speaking, the present data do not appear to support a perceptual interpretation of discrimination contrast. Insofar as the procedural differences between Groups $D$ and $E$ in the present investigation may be considered as contingent vs noncontingent reinforcement experience with larger magnitudes, the present results are consistent with a response (emotional) analysis of discrimination contrast.

\section{REFERENCES}

BOWER, G. H. A contrast effect in differential conditioning. Journal of Experimental Psychology, 1961, 62, 196-199.

DUNHAM, P. J. Contrasted conditions of reinforcement: A selective critique. Psychological Bulletin, 1968, 69, 295-315.

LUDVIGSON, H. W. \& GAY, S. E. Differential reward conditioning: Scontrast as a function of the magnitude of St. Psychonomic Science, $1966,5,289-290$.

\section{NOTES}

1. The authors are indebted to James H. McHose for advice and assistance throughout the course of the investigation which was supported by Research Grant MH 10340 from the United States Public Health Service.

2. Now at the University of South Dakota.

\section{(Continued from page 34)}

were somewhat unexpected. The number of UR sequences during acquisition was not the variable responsible for the magnitude of the PRE, although some UR sequences must be present for the PRE to occur. In the previous experiments the group with no UR sequences showed no PRE; however, when some UR sequences were present the effect occurred and some other variable must have been responsible for the magnitude of the effect.

Since percentage, number, and length of run of nonreinforcements were all confounded in the present experiment, theories emphasizing any of these variables as responsible for the magnitude of the PRE could account for the results of this experiment, although none can account for the differences between this experiment and the previous ones. Lawrence \& Festinger's (1962) dissonance theory would attribute the increased PRE to extra attractions which were developing during the increased number of nonreinforced trials, but this was not the case in the previous experiments, and when the nonreinforced trials were not followed by a reinforced one no PRE was present. Capaldi's (1966) emphasis on the length of run of nonreinforcements would explain the increased PRE in this experiment in terms of the longer length of runs of successive nonreinforcements, but again this increased PRE was not found in the previous experiments. With the small number of trials in this experiment predictions from Amsel's (1958) frustration explanation of the PRE would be indeterminate.
Since the previous conclusions were based upon a finding of no difference, the results may have been due to insensitivity, although differences were not even in the direction predicted by the other variables. Only the schedules of reinforcement were changed from the previous experiments and perhaps some subtle differences in the schedules are responsible for the magnitude of the PRE.

\section{REFERENCES}

AMSEL, A. The role of frustrative nonreward in noncontinuous reward situations. Psychological Bulletin, 1958, 55, 102-119.

CAPALDI, E. J. Partial reinforcement: A hypothesis of sequential effects. Psychological Review, 1966, 73, 459-477.

GROSSlighT, J. H., \& RADLOW, R. Patterning effect of the nonreinforcement-reinforcement sequence involving a single nonreinforced trial. Journal of Comparative \& Physiological Psychology, 1957, 50, 23-25.

KOTESKEY, R. L., \& STETTNER, L. J. Role of nonreinforcementreinforcement sequences in the partial reinforcement ef fect. Journal of Experimental Psychology, 1968. 76, 198-205.

LAWRENCE, D. H., \& FESTINGER, L. Deterrents and reinforcement. Stanford: Stanford University Press, 1962.

LEWIS, D. J. Partial reinforcement: A selective review of the literature since 1950. Psychological Bulletin, 1960, 57, 1-28. NOTES

1. This article is based on a dissertation submitted to Wayne State University in partial fulfillment of the requirements for the Ph.D. degree. The author wishes to thank Laurence Stettner for his advice and guidance during the entire investigation. The author held a NASA predoctoral traineeship grant during the investigation.

2. Now at Greenville College. 